\title{
Solutions of Generalized ARE by Hamiltonian Pencils
}

\author{
Fan-Ren Chang ${ }^{\dagger}$ and Chang-Chun Chen \\ Department of Electrical Engineering \\ National Taiwan University \\ Taipei, Taiwan, 10764 \\ Republic of China
}

\begin{abstract}
To solve the infinite-horizon linear quadratic regulation design for generalized systems $E \dot{x}(t)=A x(t)+B u(t)$, Lewis (1985) has developed the generalized algebraic Riccati equation (GARE). In order to obtain an analytic solution of GARE, the Hamiltonian pencil is introduced in this note. Eigenvectors of the Hamiltonian pencil play the key role in solving the mentioned problem. Our approach is a nature extension of the optimal control in regular linear systems.
\end{abstract}

Key Words - Linear Quadratic Regulator, Generalized System, Matrix Pencil

\section{Introduction}

Generalized state-space systems or singular systems or descriptor systems have received much attention (Verghese et al. (1981), Lewis (1986)) due to extensive applications in large-scale interconnected systems such as power systems, robotic manipulators, economics systems and circuits systems. The problem of the linear-quadratic optimal control for the generalized systems is discussed in many papers also (Bender et al. (1987), Lewis (1985), Wang (1991)). It is well known that the algebraic Riccati equation (ARE) has played the key role in the design of the infinite-horizon linear-quadratic regulator (LQR) for regular linear systems $\dot{x}(t)=A x(t)+B u(t)$. In order to find the appropriate state feedback gain of LQR, those stable eigenvectors of the Hamiltonian matrix corresponded to the given ARE can provide the analytical solution. ARE has been extended to GARE (generalized algebraic Riccati equation) by Lewis (1985) to solve the infinite-horizon linear-quadratic regulator problem for generalized linear systems $E \dot{x}(t)=A x(t)+B u(t)$, in which $E$ may be singular. But, solutions of GARE were not proposed by him. Arnold et al. (1984) and Lin (1989) have investigated numerical solutions of GARE, however, their works are restricked to the case of $E$ being nonsingular, which is not the common situation of generalized systems. In this note, we extend the Hamiltonian matrix to a linear matrix pencil (named as Hamiltonian pencil), whose eigenvectors will solve the GARE.

In section II, we briefly introduce some fundamental concepts of generalized systems as well as some important results of Lewis (1985). In section III, the properties of the Hamiltonian pencil corresponded to the GARE are investigated. From which, the solutions of GARE and the optimal gain can be derived. A numerical example has been provided in section IV. Finally, conclusions are stated in section $\mathrm{V}$.

\section{Preliminaries}

Let's consider the generalized system

$$
E \dot{x}(t)=A x(t)+B u(t)
$$

where $x \in R^{n}, u \in R^{m}, E, A \in R^{n \times n}$ and $B \in R^{n \times m}$. $E$ may be a singular matrix. The linear pencil $(s E-A)$ is assumed to be regular (i.e. $\operatorname{det}(s E-A) \not \equiv 0$ ).

† To whom all correspondence should be addressed. 0-7803-0510-8/92\$03.00 @1992IEEE
The following terms describe basic properties of generalized systems, which are defined by Lewis (1985).

Definition 1 The triple $\{E, A, B\}$ is controllable if the controllability pencil

$$
\left[\begin{array}{ll}
s E-A & B
\end{array}\right]
$$

has no finite or infinite zeros.

Definition 2 The triple $\{E, A, B\}$ is stabilizable if the controllability pencil (2) has no infinite zeros and all finite zeros of (2) are in the open left-half plane.

Definition 3 The triple $\{E, A, C\}$ is observable if the observability pencil

$$
\left[\begin{array}{c}
s E-A \\
C
\end{array}\right]
$$

has no finite or infinite zeros.

Definition 4 The pair $\{E, A\}$ is asymptotically stable (AS) if all roots of $\Delta(s)=\operatorname{det}(s E-A)=0$ are in the open left half plane.

Definition 5 The pair $\{E, A\}$ is impulse free if (1) exhibits no impulse behavior. This is equivalent to the absence of infinites zeros of $(s E-A)$.

Remark 1 There are several other equivalent descriptions of impulse free. For examples, $\{E, A\}$ is impulse-free means $(s E-A)^{-1}$ is a proper rational matrix, or there does not exist any chained infinite eigenstructure in $(s E-A)$.

The infinite-horizon linear-quadratic regulator problem of generalized system (1) is to find the optimal control $u^{*}(t)$ such that the performance index

$$
J\left(E x_{0}, u(\cdot)\right)=\int_{0}^{\infty}\left[x^{T}(t) Q x(t)+u^{T}(t) R u(t)\right] d t
$$

is minimized, where $R=R^{T}>0, Q=Q^{T} \geq 0$ and $x_{0}$ is the initial state of (1) at time 0 . Lewis(1985) has derived the optimal control law

$$
u^{*}(t)=-R^{-1} B^{T} P E x(t)
$$

where $P$ is the positive definite solution of the generalized algebraic Riccati equation (GARE)

$$
E^{T} P A+A^{T} P E-E^{T} P B R^{-1} B^{T} P E+Q=0
$$

under the conditions described in the following:

1. $\{E, A\}$ is impulse free.

2. $\{E, A, B\}$ is stabilizable.

3. $\{E, A, C\}$ is observable where $C$ is decomposed from the weighting matrix $Q$ by $Q=E^{T} C^{T} C E$.

Also, Lewis (1985) has indicated that the closed-loop system under optimal gain of (5) will be asymptotically stable and impulse free.

It is quite obvious, solution of (6) is necessary to accomplish the optimal control law. One more point, $P E$ will be enough to compute $u^{*}(t)$ in (5). Comparing PE in GARE (6) with $\widetilde{P}$ in ARE (7) 


$$
P A+A^{T} P-P B R^{-1} B^{T} P+Q=0
$$

there exist two major differences:

1. $P E$ is not necessary symmetric but $\bar{P}$ is always symmetric.

2. $P E$ is always singular if $E$ is singular but $\bar{P}$ is not necessary singular.

Hence, to develop a method for solving GARE is an interesting problem. We will discuss it in the next section.

\section{Main results}

Hamiltonian matrix

$$
\left[\begin{array}{cc}
A & -B R^{-1} B^{T} \\
-Q & -A^{T}
\end{array}\right]
$$

corresponded to (7) has a great help in solving ARE. Hence, we construct the following pencil

$$
s F-H \triangleq s\left[\begin{array}{cc}
E & 0 \\
0 & E^{T}
\end{array}\right]-\left[\begin{array}{cc}
A & -B R^{-1} B^{T} \\
-Q & -A^{T}
\end{array}\right]
$$

and call it as the Hamiltonian pencil. It will be shown later that the eigenvectors of Hamiltonian pencil can solve GARE (6). Some properties of $s \boldsymbol{F}-H$ are discussed first.

Lemma If $\lambda$ is a finite eigenvalue of the Hamiltonian pencil (9), then $-\lambda$ is also a finite eigenvalue of it.

Proof: Let $\left[\begin{array}{l}v_{1} \\ v_{2}\end{array}\right]$ be the eigenvector associated to $\lambda$.

Then

$$
\left\{\lambda\left[\begin{array}{cc}
E & 0 \\
0 & E^{T}
\end{array}\right]-\left[\begin{array}{cc}
A & -B R^{-1} B^{T} \\
-Q & -A^{T}
\end{array}\right]\right\}\left[\begin{array}{l}
v_{1} \\
v_{2}
\end{array}\right]=0
$$

It is equivalent to

$$
\begin{aligned}
\lambda E v_{1} & =A v_{1}-B R^{-1} B^{T} v_{2} \\
\lambda E^{T} v_{2} & =-Q v_{1}-A^{T} v_{2}
\end{aligned}
$$

Take transpose on both sides

$$
\begin{aligned}
\lambda v_{1}^{T} E^{T} & =v_{1}^{T} A^{T}-v_{2}^{T} B R^{-1} B^{T} \\
\lambda v_{2}^{T} E & =-v_{1}^{T} Q-v_{2}^{T} A
\end{aligned}
$$

They can be arranged into the matrix form

$$
\begin{aligned}
& -\lambda\left[\begin{array}{ll}
-v_{2}^{T} & v_{1}^{T}
\end{array}\right]\left[\begin{array}{cc}
E & 0 \\
0 & E^{T}
\end{array}\right] \\
& =\left[\begin{array}{ll}
-v_{2}^{T} & v_{1}^{T}
\end{array}\right]\left[\begin{array}{cc}
A & -B R^{-1} B^{T} \\
-Q & -A^{T}
\end{array}\right]
\end{aligned}
$$

It is equivalent to

$$
\begin{aligned}
{\left[\begin{array}{ll}
-v_{2}^{T} & v_{1}^{T}
\end{array}\right]\left\{-\lambda\left[\begin{array}{cc}
E & 0 \\
0 & E^{T}
\end{array}\right]-\left[\begin{array}{cc}
A & -B R^{-1} B^{T} \\
-Q & -A^{T}
\end{array}\right]\right\} } \\
=\left[\begin{array}{ll}
0 & 0
\end{array}\right]
\end{aligned}
$$

Hence $-\lambda$ is also a finite eigenvalue of $(s F-H)$.

Remark 2 Under the conditions (i) $\{E, A\}$ impulse free, (ii) $\{E, A, B\}$ stabilizable and (iii) $\{E, A, C\}$ observable just as described in the previous section, Lewis (1985) has shown that the optimal closed-loop system is asymptotically stable, whose finite poles are those left half-plane eigenvalues of the Hamiltonian pencil. Also, the result of optional control is impulse free. It means that there exist $q(\triangleq$ rank $E)$ finite eigenvalues and $(n-q)$ infinite unchained eigenvalues in the closed-loop system. Hence, there are $2 q$ finite eigenvalues and $2(n-q)$ infinite unchained eigenvalues associated to the Hamiltonian pencil.

Note that $(s F-H)$ and $(s H-F)$ have the same set of eigenvectors but their eigenvalues have the reciprocal relationship. Hence, infinite eigenvalues of $(s F-H)$ become zero eigenvalues of $(s H-F)$. Instead of considering $(s F-H)$, we investigate the pencil $(s H-F)$. Let's assume the eigenstructure of $(s H-F)$

$$
\begin{aligned}
& {\left[\begin{array}{cc}
A & -B R^{-1} B^{T} \\
-Q & -A^{T}
\end{array}\right]\left[\begin{array}{ll}
T_{11} & T_{12} \\
T_{21} & T_{22}
\end{array}\right]\left[\begin{array}{cc}
A & 0 \\
0 & -\Lambda
\end{array}\right]} \\
& =\left[\begin{array}{cc}
E & 0 \\
0 & E^{T}
\end{array}\right]\left[\begin{array}{ll}
T_{11} & T_{12} \\
T_{21} & T_{22}
\end{array}\right]
\end{aligned}
$$

where $\left[\begin{array}{ll}T_{11} & T_{12} \\ T_{21} & T_{22}\end{array}\right],\left[\begin{array}{cc}\Lambda & 0 \\ 0 & -\Lambda\end{array}\right]$ are constructed by the eigenvectors and eigenvalues respectively, and $\Lambda$ contains $q$ finite left half-plane eigenvalues and $(n-q)$ zero eigenvalues. From (10), we get

$$
A T_{11} \Lambda-B R^{-1} B^{T} T_{21} \Lambda=E T_{11}
$$

$$
-Q T_{11} \Lambda-A^{T} T_{21} \Lambda=E^{T} T_{21}
$$

If $T_{11}$ is invertible then

$$
\begin{aligned}
A T_{11} A T_{11}^{-1}-B R^{-1} B^{T} T_{21} A T_{11}^{-1} & =E \\
-Q T_{11} A T_{11}^{-1}-A^{T} T_{21} T_{11}^{-1} T_{11} A T_{11}^{-1} & =E^{T} T_{21} T_{11}^{-1}
\end{aligned}
$$

Set

$$
\begin{aligned}
& Y \triangleq T_{11} \Lambda T_{11}^{-1} \\
& X \triangleq T_{21} T_{11}^{-1}
\end{aligned}
$$

Then (12) can be simplified as

$$
\begin{aligned}
\left(A-B R^{-1} B^{T} X\right) Y & =E \\
\left(-Q-A^{T} X\right) Y & =E^{T} X
\end{aligned}
$$

Let (15) be left multiplied by $X^{T}$,

$$
\left(X^{T} A-X^{T} B R^{-1} B^{T} X\right) Y=X^{T} E
$$

(17) subtracted from (16) becomes

$$
\left(X^{T} A+A^{T} X-X^{T} B R^{-1} B^{T} X+Q\right) Y=X^{T} E-E^{T} X
$$

Comparing (18) with GARE (6) it can be easily checked that if we set $X=P E$, then the right side of $(18)$ is $(P E)^{T} E-E^{T} P E=$ 0. Hence, (18) becomes

$$
\left(E^{T} P A+A^{T} P E-E^{T} P B R^{-1} B^{T} P E+Q\right) Y=0
$$

where $P E=T_{21} T_{11}^{-1}$ as defined in (14).

(19) tells us that $T_{21} T_{11}^{-1}$ is a necessary solution $P E$ of GARE. Since $Y \triangleq T_{11} \Delta T_{11}^{-1}$ will be a singular matrix if $E$ is singular, we can not remove $Y$ from (19). In order to make sure the answer is correct, we need check the result by substitute $T_{21} T_{11}^{-1}$ into (6). The optimal gain then will be $-R^{-1} B^{T} T_{21} T_{11}^{-1}$ as shown in (5).

At the end of this section, the computational procedures for generalized systems linear-quadratic regulator design are summarized below.

Step 1: Construct the Hamiltonian pencil $(s F-H)$ from $E$, $A, B$ and $Q, R$ as shown in (9).

Step 2: Find the eigenstrure of $(s H-F)$ as shown in (10).

Step 3: Substitue $P E$ by $T_{21} T_{11}^{-1}(14)$ into (6), to verify the solution.

Step 4: The optimal control $u^{*}(t)=-R^{-1} B^{T} T_{21} T_{11}^{-1} x(t)$.

\section{Example}

One numerical example is given to show our computational procedure for the optimal control.

Example:

Given

$$
E \dot{x}(t)=A x(t)+B u(t), \quad J=\int_{0}^{\infty}\left[x^{T}(t) Q x(t)+u^{T}(t) R u(t)\right] d t
$$

where 


$$
\begin{aligned}
& E=\left[\begin{array}{llll}
1 & 0 & 0 & 0 \\
0 & 0 & 1 & 0 \\
0 & 0 & 0 & 0 \\
0 & 0 & 0 & 0
\end{array}\right], \quad A=\left[\begin{array}{cccc}
0 & 1 & 0 & 0 \\
0 & 0 & 0 & 1 \\
1 & 1 & 0 & 0 \\
0 & -1 & 1 & 1
\end{array}\right] . \\
& B=\left[\begin{array}{ll}
0 & 0 \\
0 & 0 \\
1 & 0 \\
0 & 1
\end{array}\right], \quad C=\left[\begin{array}{llll}
0 & 1 & 0 & -1 \\
0 & 0 & 0 & 1
\end{array}\right] . \\
& Q=E^{T} C^{T} C E=\left[\begin{array}{llll}
0 & 0 & 0 & 0 \\
0 & 0 & 0 & 0 \\
0 & 0 & 1 & 0 \\
0 & 0 & 0 & 0
\end{array}\right], \quad R=\left[\begin{array}{ll}
1 & 0 \\
0 & 1
\end{array}\right] .
\end{aligned}
$$

Find the optimal control law.

\section{Solution:}

Construct the Hamiltonian pencil $(s F-H)$, where

$$
\begin{aligned}
F & =\left[\begin{array}{cc}
E & 0 \\
0 & E^{T}
\end{array}\right] \\
& =\left[\begin{array}{cccccccc}
1 & 0 & 0 & 0 & 0 & 0 & 0 & 0 \\
0 & 0 & 1 & 0 & 0 & 0 & 0 & 0 \\
0 & 0 & 0 & 0 & 0 & 0 & 0 & 0 \\
0 & 0 & 0 & 0 & 0 & 0 & 0 & 0 \\
0 & 0 & 0 & 0 & 1 & 0 & 0 & 0 \\
0 & 0 & 0 & 0 & 0 & 0 & 0 & 0 \\
0 & 0 & 0 & 0 & 0 & 1 & 0 & 0 \\
0 & 0 & 0 & 0 & 0 & 0 & 0 & 0
\end{array}\right] \\
& =\left[\begin{array}{cccccccc}
A & -B R^{-1} & B^{T} \\
-Q & & -A^{T} & & &
\end{array}\right. \\
& =\left[\begin{array}{cccccccc}
0 & 1 & 0 & 0 & 0 & 0 & 0 & 0 \\
0 & 0 & 0 & 1 & 0 & 0 & 0 & 0 \\
1 & 1 & 0 & 0 & 0 & 0 & -1 & 0 \\
0 & -1 & 1 & 1 & 0 & 0 & 0 & -1 \\
0 & 0 & 0 & 0 & 0 & 0 & -1 & 0 \\
0 & 0 & 0 & 0 & -1 & 0 & -1 & 1 \\
0 & 0 & -1 & 0 & 0 & 0 & 0 & -1 \\
0 & 0 & 0 & 0 & 0 & -1 & 0 & -1
\end{array}\right]
\end{aligned}
$$

The eigenvetors and eigenvalues matrices of the pencil $(s \mathrm{H}-\mathrm{F})$ are

$$
\begin{aligned}
V & =\left[\begin{array}{ll}
T_{11} & T_{12} \\
T_{21} & T_{22}
\end{array}\right] \\
& {\left[\begin{array}{ccccccccc}
0.1196 & -0.5152 & 0 & 0 & 0.2053 & -0.3067 & 0 & 0 \\
-0.2211 & 0.3947 & -1 & 0 & 0.3793 & -0.2347 & 0 & 0 \\
0.4451 & 0.4922 & 0 & 0 & 0.2274 & -0.0389 & 0 & 0 \\
-0.8225 & -0.3767 & 0 & 1 & 0.4202 & -0.0298 & 0 & 0 \\
& & & & & & & \\
-0.0549 & -0.1579 & 0 & 0 & -0.3164 & 0.7073 & 0 & 0 \\
0.1563 & 0.2788 & 0 & 0 & -0.2682 & -0.1660 & 0 & 0 \\
-0.1014 & -0.1209 & 0 & 0 & 0.5846 & -0.5414 & 1 & 0 \\
-0.1563 & -0.2788 & 0 & 0 & 0.2682 & 0.1660 & 0 & 1
\end{array}\right] }
\end{aligned}
$$

$$
\begin{aligned}
D & =\left[\begin{array}{cc}
\Lambda & 0 \\
0 & -\Lambda
\end{array}\right] \\
& =\left[\begin{array}{cccccccc}
\frac{1}{-1.8478} & 0 & 0 & 0 & 0 & 0 & 0 & 0 \\
0 & \frac{1}{-0.7654} & 0 & 0 & 0 & 0 & 0 & 0 \\
0 & 0 & 0 & 0 & 0 & 0 & 0 & 0 \\
0 & 0 & 0 & 0 & 0 & 0 & 0 & 0 \\
0 & 0 & 0 & 0 & \frac{1}{1.8478} & 0 & 0 & 0 \\
0 & 0 & 0 & 0 & 0 & \frac{1}{0.7654} & 0 & 0 \\
0 & 0 & 0 & 0 & 0 & 0 & 0 & 0 \\
0 & 0 & 0 & 0 & 0 & 0 & 0 & 0
\end{array}\right]
\end{aligned}
$$

Take

$$
\begin{aligned}
& T_{11}=\left[\begin{array}{cccc}
0.1196 & -0.5152 & 0 & 0 \\
-0.2211 & 0.3947 & -1 & 0 \\
0.4451 & 0.4922 & 0 & 0 \\
-0.8225 & -0.3767 & 0 & 1
\end{array}\right] \\
& T_{21}=\left[\begin{array}{cccc}
-0.0549 & -0.1579 & 0 & 0 \\
0.1563 & 0.2788 & 0 & 0 \\
-0.1014 & -0.1209 & 0 & 0 \\
-0.1563 & -0.2788 & 0 & 0
\end{array}\right] \\
& \text { Substitue } P E=T_{21} T_{11}^{-1}=\left[\begin{array}{cccc}
0.1502 & 0 & -0.1637 & 0 \\
-0.1637 & 0 & 0.3951 & 0 \\
0.0135 & 0 & -0.2315 & 0 \\
0.1637 & 0 & -0.3951 & 0
\end{array}\right] \text { into }
\end{aligned}
$$

(6) to verify the result being zero.

Then the feedback gain

$$
K=R^{-1} B^{T} P E=\left[\begin{array}{llll}
0.0137 & 0 & -0.2315 & 0 \\
0.1637 & 0 & -0.3951 & 0
\end{array}\right]
$$

and the optimal control is $u^{*}(t)=-K x(t)$. The colsed-loop system will be

$$
\left[\begin{array}{llll}
1 & 0 & 0 & 0 \\
0 & 0 & 1 & 0 \\
0 & 0 & 0 & 0 \\
0 & 0 & 0 & 0
\end{array}\right] \dot{x}(t)=\left[\begin{array}{cccc}
0 & 1 & 0 & 0 \\
0 & 0 & 0 & 1 \\
0.9865 & 1 & 0.2315 & 0 \\
-0.1637 & -1 & 1.3951 & 1
\end{array}\right] x(t)
$$

Its finite pole are $s_{1}=-1.8478$ and $s_{2}=-0.7654$ which are coincide with those stable eigenvalues of Hamiltonian pencil as shown in $D$ matrix.

\section{Conclusion}

In this paper we provide an analytic solution to generalized systems infinite-horizon linear-quadratic regulator problem. The solution of GARE is obtained from the eigeustructure of the Hamiltonian pencil. If the $\mathrm{E}$ matrix happens to be identity, then our result become the typical method for regular linear-quadratic design as in Anderson et al.(1989). Bender et al.(1987) attack the same problem by coordinate transformation to separate the finite and infinite subsystems. The requirement of the given plant being impulse free is not needed in their work. But, all interesting compatible properties, such as GARE vs. ARE, Hamiltonian pencil vs. Hamiltonian matrix, $E$ matrix vs. $I$ matrix can not be found in Bender's(1987) paper due to the destroyed structure caused by bases changing.

In the derivation of analytic solution derivation, numerical reliable consideration is not discussed. Arnold et al.(1984) recommended the numerical stabled Schur method to solve ARE. To adopt the orthogonal Schur method in computation the eigenspace of Hamiltonian pencil needs further studies.

\section{References}

[1] B. D. O. Anderson and J. B. Moore (1990). Optimal Control-Linear Quadratic Methods. Prentice Hall, Englewood Cliffs, New Jersey.

[2] W. F. Arnold and A. J. Laub (1984). Generalized eigenproblem algorithms and software for algebraic Riccati equestions. Proc. IEEE, 72, 12, 1746-1754.

[3] D. J. Bender and A. J. Laub (1987). The linear quadratic optimal regulator for descriptor systems. IEEE Trans Automatic Control, AC-32, 8, 672-688.

[4] F. L. Lewis (1985) Preliminary notes on optimal control for singular systems. Proc. 24th Conf. Decision Control, 266272.

[5] F. L. Lewis (1986) A suruey of linear singular systems. Circuits Systems Signal Process, 5 , 3-36. 
[6] W. W. Lin (1989) An SDR algorithm for the solution of the generalized algebraic Riccati equation. IEEE Trans Automatic Contiol, AC-34, 8, 875-879

[7] E. Wang (1991) Local maximum principle for the singular linear systems. Recent Advances in Mathematical Theory of Systems, Control, Networks and Signal Processing Proceeding II (Proc. of the International Symposium MTNS-91), 103-108. 